ROCKY MOUNTAIN

JOURNAL OF MATHEMATICS

Volume 21, Number 2, Spring 1991

\title{
A HYPERBOLIC STEFAN PROBLEM
}

\author{
R.E. SHOWALTER AND N.J. WALKINGTON
}

1. Introduction. In order to utilize the balance of energy to calculate temperatures, it is necessary to introduce constitutive assumptions relating to energy storage, $e$, and flux, $\vec{q}$, to the temperature $u$. Traditionally, $e$ is chosen proportional to $u$ and $\vec{q}$ proportional to $\nabla u$. This leads to the classical parabolic heat equation, where a thermal disturbance is instantly felt throughout the body. Other admissible constitutive relations allow $e$ and $q$ to depend upon the temperature history [9],

$$
\vec{q}(t)=-\int_{0}^{\infty} a(s) \nabla u(t-s) d s .
$$

Selecting $a(s)=(k / \tau) e^{-(s / \tau)}$ in the above (cf. [9]) yields

$$
\left(1+\tau \frac{d}{d t}\right) \vec{q}=-k \nabla u
$$

which leads to the hyperbolic telegraphers equation

$$
\tau c u_{t t}+c u_{t}-k \Delta u=0
$$

in place of the classical heat equation $(\tau=0)$. Such a model will certainly have an upper bound on the speed of thermal disturbances.

We shall formulate a well-posed free-boundary problem of the Stefan type $[\mathbf{7 , 1 3}-\mathbf{1 5}]$ consistent with (1.1) and which contains the telegraphers equation (1.2).

2. Energy and phase change models. If a block of ice of unit volume is subject to a uniform heat source of intensity $F>0$, classical models predict a temperature increase at the rate of $F / c_{1}$ until the temperature reaches $u=0$. While the ice melts the temperature remains at zero and the fraction of water, $\xi$, increases from 0 to 1 at a

Received by the editors on April 12, 1988.

This work was supported by a grant from the National Science Foundation.

Copyright (1991 Rocky Mountain Mathematics Consortium 
rate of $F / L$ where $L$ is the latent energy of fusion. When all of the ice has melted the temperature increases at a rate of $F / c_{2}$. The constants $c_{1}$ and $c_{2}$ are the specific heats of the ice and water, respectively. In summary, the energy constant is given by

$$
e=C(u)+L \xi
$$

where $C(u)=c_{1} u$ for $u<0, C(u)=c_{2} u$ for $u \geq 0$ and $\xi \in H(u)$, the Heaviside graph of $u$, is the water fraction. The classical model (2.1) assumes that the storage and release of heat is instantaneous, this being compatible with the assumption that the flux is proportional to the temperature gradient.

We consider here a constitutive energy relation

$$
\left(1+\tau \frac{d}{d t}\right) e=\left(1+T(u) \frac{d}{d t}\right) C(u)+L \xi, \quad \xi \in H(u)
$$

where $T(u)=\tau_{1} u$ if $u<0$ and $T(u)=\tau_{2} u$ if $u \geq 0$. Equation (2.2) may be motivated from a memory dependent model as indicated for the heat flux $\vec{q}$ in (1.1). The "delay times" $\tau_{1}$ and $\tau_{2}$ will be chosen to match wave speeds in the solid and liquid phases. Repeating the above experiment using (2.2) for an energy storage model gives the same temperature rise rate $F / c_{1}$ until $u=0$. Then $\xi$ jumps from 0 to $\tau_{1} F / L$; thereafter, it increases steadily to 1 where $e=L-\tau F$. Afterward, temperature increases again, given by

$$
u=\left(\frac{1}{c_{2}}\right)\left\{e-(L-\tau F)-F \tau_{2}\left(1-\exp \left[\frac{-e+L-\tau F}{\tau_{2} F}\right]\right) L\right\} .
$$

The nonclassical feature observed here is that a fraction of water melts instantly when temperature reaches zero. Note that $\tau_{1}$ must be small, $\tau_{1} F \ll L$, for the preceding, and there is a smoothing of the classical temperature response near the end of the melting interval at the expense of the discontinuity in latent heat stored at the beginning of this interval.

3. The Stefan problem. The balance of energy and constitutive relations (1.1) and (1.2) will be used to formulate a Stefan problem containing the telegraphers equation. This problem is naturally posed 
in a Hilbert space of distributions. Let $G$ be a domain in Euclidean space $\mathbf{R}^{m}$ and set $\Omega=G \times(0, \infty)$. The temperature at point $x \in G$ and time $t>0$ is $u(x, t)$; from the conductivities of ice and water, $k_{1}$ and $k_{2}$, respectively, we define the function $K$ by $K(u)=k_{1} u$ for $u \leq 0$ and $K(u)=k_{2} u$ for $u \geq 0$. Recall that $C(u)$ and $T(u)$ are determined similarly from specific heats and delay times. Denote by $\Omega_{-}, \Omega_{+}$and $\Omega_{0}$ the subdomains of $\Omega$ where $u<0, u>0$ and $u=0$, respectively. Let $S_{+}$be the boundary of $\Omega_{+}$and $S_{-}$the boundary of $\Omega_{-}$in $\Omega . N=\left(N_{t}, N_{x}\right)$ is the normal on either $S_{+}$or $S_{-}$oriented out of $\Omega_{+}$and into $\Omega_{-}$, respectively, so that it is assigned consistently on $S \equiv S_{+} \cap S_{-}$, the interface between $\Omega_{+}$and $\Omega_{-}$. Hereafter, we assume that the flux and energy are related to temperature by (1.1) and (2.2), respectively.

The balance of energy requires that for any subdomain $G^{*} \subset G$ with outward normal $\vec{n}$

$$
\frac{d}{d t} \int_{G^{*}}\left(e+\tau e^{\prime}\right)=-\int_{\partial G^{*}}\left(\vec{q}+\tau \vec{q}^{\prime}\right) \cdot \vec{n}+\int_{G^{*}}\left(F+\tau F^{\prime}\right) .
$$

The form of our constitutive relations suggests that the integrands will be piecewise smooth in $\Omega$. Writing the integrals as sums over the smooth portions and their boundaries $\tilde{S}$ and utilizing the Leibnitz rule to bring the temporal derivative under the integral enables one to compute

$$
\begin{aligned}
\int_{G^{*}} & \left\{\frac{\partial}{\partial t}\left(e+\tau e^{\prime}\right)+\nabla \cdot\left(\vec{q}+\tau \vec{q}^{\prime}\right)\right\} \\
= & \int_{G^{*}}\left(F+\tau F^{\prime}\right)+\int_{\tilde{S} \cap G^{*}}\left[e+\tau e^{\prime}\right] V(t)+\left[\vec{q}+\tau \vec{q}^{\prime}\right] \cdot \vec{n} .
\end{aligned}
$$

The formulation of a Stefan problem for processes whose smoothness is consistent with our constitutive relations follows from the combination of (1.1), (2.2) and (3.1). The problem is to find functions $u, \xi$ on 
$\Omega$ such that $\xi \in H(u)$ and

$$
\frac{\partial}{\partial t}\left(\tau_{2} c_{2} \frac{\partial u}{\partial t}+c_{2} u\right)-k_{2} \Delta u=F+\tau F^{\prime} \quad \text { in } \Omega_{+}-\tilde{S},
$$

$$
\begin{aligned}
& L \frac{\partial \xi}{\partial t}=F+\tau F^{\prime} \quad \text { in } \Omega_{0}-\tilde{S} \\
& \frac{\partial}{\partial t}\left(\tau_{1} c_{1} \frac{\partial u}{\partial t}+c_{1} u\right)-k_{1} \Delta u=F+\tau F^{\prime} \quad \text { in } \Omega_{-}-\tilde{S}
\end{aligned}
$$

$$
\left(L(1-\xi)+\tau_{2} c_{2} \frac{\partial u}{\partial t}\right) N_{t}=k_{2} \vec{\nabla} u \cdot N_{x} \quad \text { on } S_{+},
$$$$
\left(-L \xi+\tau_{1} c_{1} \frac{\partial u}{\partial t}\right) N_{t}=k_{1} \vec{\nabla} u \cdot N_{x} \quad \text { on } S_{-},
$$$$
\tau_{i} c_{i}\left[\frac{\partial u}{\partial t}\right] N_{t}=k_{i}[\nabla u] N_{x} \quad \text { on } \tilde{S}-S,
$$

where $i=1$ or 2 depending upon $u$ being $<0$ or $>0$,

$$
u(s, t)=0, \quad s \in \partial G, t>0,
$$

$$
u(x, 0)=u_{0}(x), \quad x \in G,
$$

$$
\left.\left(\tau_{2} c_{2} \frac{\partial u}{\partial t}+c_{2} u+L\right)\right|_{t=0}=v_{0}(x) \quad \text { where } u_{0}(x)>0
$$

$$
\left.\left(\tau_{1} c_{1} \frac{\partial u}{\partial t}+c_{2} u\right)\right|_{t=0}=v_{0}(x) \quad \text { where } v_{0}(x)<0
$$

$$
L \xi\left(x, 0^{+}\right)=v_{0}(x) \quad \text { where } u_{0}(x)=0, x \in G .
$$

The temperature in the water and ice is governed by the hyperbolic telegraphers equations (3.2a) and (3.2c), respectively, and the water 
fraction in the partially frozen region is determined by the ordinary differential equation (3.2b). Equations (3.3a) and (3.3b) determine the free surface (at $u=0$ ) in the water and ice, respectively. Where $\Omega_{0}$ has zero measure the jump conditions (3.3a) and (3.3b) combine to give the jump in values along $S=S_{+} \cap S_{-}$,

$$
\left(L+\tau_{0}\left[\frac{d}{d t} K(u)\right]\right) N_{t}=\left[\nabla K(u) \cdot N_{x}\right] \quad \text { on } S .
$$

This is the counterpart of the usual Stefan constraint $\left(\tau_{0}=0\right)$. Also (3.3c) is the classical jump condition along wave fronts. Any of the classical boundary conditions could be used in place of (3.3d). The initial conditions $u=u_{0}$ and $\left(e+\tau e^{\prime}\right)=v_{0}$ are given in (3.4). Nonlinearities arise from the free surface and the difference in material properties $c_{i}, k_{i}$ and $\tau_{i}$ for water $(i=1)$ and ice $(i=2)$. Note that the speeds of propagation of disturbances along the characteristics are given by $\left(k_{2} / c_{2} \tau_{2}\right)^{1 / 2}$ and $\left(k_{1} / c_{1} \tau_{1}\right)^{1 / 2}$ in the respective phases. We shall require that there be a global signal speed independent of phase; to fix the ratio of $\tau_{1}$ and $\tau_{2}$ to obtain a single velocity equal to $\left(1 / \tau_{0}\right)^{1 / 2}$ with $\tau_{0}>0$ we choose

$$
\tau_{1}=\frac{\tau_{0} k_{1}}{c_{1}}, \quad \tau_{2}=\frac{\tau_{0} k_{2}}{c_{2}}
$$

everywhere above.

Under the same smoothness assumptions used to derive the balance of energy statement (3.1), a computation shows that (3.2) and (3.3) are equivalent to

$$
\frac{\partial}{\partial t}\left(\tau_{0} \frac{\partial K(u)}{\partial t}+C(u)+L \xi\right)-\Delta K(u)=F+\tau F^{\prime}
$$

in $\mathcal{D}^{\prime}(\Omega)$. A generalized solution of the Stefan problem (3.2)-(3.4) is a pair of functions

$$
u \in W^{1, \infty}\left(0, T ; L^{2}(G)\right) \bigcap L^{\infty}\left(0, T ; H_{0}^{1}(G)\right), \quad \xi \in L^{\infty}(\Omega)
$$


which satisfy

$$
\begin{gathered}
\frac{d}{d t}\left(\tau_{0} \frac{d}{d t} K(u(t))+C(u(t))+L \xi(t)\right)-\Delta K(u(t)) \\
=F+\tau F^{\prime}, \quad \text { a.e. } t \in[0, T]
\end{gathered}
$$

$$
u(t) \in H_{0}^{1}(G) \text { and } \xi(t) \in H(u(t)) \text { for all } t \in[0, T],
$$

$$
u(0)=u_{0}, \quad\left(\tau_{0} \frac{d}{d t} K(u)+C(u)+L \xi\right)(0)=v_{0} .
$$

It will always be assumed that $F \in W^{1, \infty}\left(0, T ; H^{-1}(G)\right)$ so it is implicit in (3.5a) that the term " $\tau_{0}(d / d t) K(u)(t)+C(u)+L \xi(t)$ " belongs to $W^{1, \infty}\left(0, T ; H^{-1}(G)\right)$. Hence, the initial conditions are meaningful.

4. Existence-uniqueness of a generalized solution. Theorem 1 below will be used to demonstrate that problem (3.5) is an evolution equation with solutions determined by a semi-group of contractions on a Hilbert space.

Theorem 1. Let $V$ and $H$ be Hilbert spaces with $V$ dense and continuously imbedded in $H$. Denote by $\mathcal{A}: V \rightarrow V^{\prime}$ and $\mathcal{C}: H \rightarrow H^{\prime}$ the Riesz maps onto their respective dual spaces and let $\mathcal{B}: V \rightarrow V^{\prime}$ be a (possibly multi-valued) maximal monotone operator. Assume $f \in W^{1,1}\left(0, T ; V^{\prime}\right), u_{0} \in V$ and $v_{0} \in V^{\prime}$ are given such that there is $a b \in \mathcal{B}\left(u_{0}\right)$ with $b-v_{0} \in H^{\prime}$. Then there exists a unique pair $u \in$ $W^{1, \infty}(0, T ; H), w \in L^{\infty}\left(0, T ; V^{\prime}\right)$ such that $\mathcal{C} u^{\prime}+w \in W^{1, \infty}\left(0, T ; V^{\prime}\right)$, $w(t) \in \mathcal{B}(u(t))$ for all $t \in[0, T]$,

$$
\left(\mathcal{C} u^{\prime}+w\right)^{\prime}+\mathcal{A}(u)=f \quad \text { in } L^{\infty}\left(0, T ; V^{\prime}\right),
$$

and $u(0)=u_{0},\left(\mathcal{C} u^{\prime}+w\right)(0)=v_{0}$.

It remains to recover the weak form of the Stefan problem (3.5) as a special case of (4.1). Make the change of variable $U=K(u)$ and observe that $H(U)=H(u)$. Then choose $H=L^{2}(G) \cong H^{\prime}$ and $\mathcal{C} u=\tau c u$ for 
$u \in H$. Similarly, let $V=H_{0}^{1}(G)$ and define $\mathcal{A} \in \mathcal{L}\left(V, V^{\prime}\right)$ by the scalar product,

$$
\mathcal{A} U(v)=\int_{G} \nabla U \cdot \nabla v, \quad U, v \in H_{0}^{1}(G),
$$

so $\mathcal{A}=-\Delta$ is a distribution-valued Laplace operator. Finally, we define $\mathcal{B}(U)=C \circ K^{-1}(U)+L H(U)$, the indicated monotone operator obtained from the $L^{2}$-realization of the (multi-valued) maximal monotone graph $C \circ K^{-1}(\cdot)+L H(\cdot)$. That is, $w \in \mathcal{B}(U)$ if and only if $w=C \circ K^{-1}(U)+L \xi=\tau_{0} u+L \xi$ with $U=K(u)$ and $\xi \in H(u)$ a.e. in $G$.

Theorem 2. Assume that $F \in W^{2,1}\left(0, T ; H^{-1}(G)\right), u_{0} \in H_{0}^{1}(G)$, $\xi_{0} \in L^{\infty}(G)$ with $\xi_{0}(x) \in H\left(u_{0}(x)\right)$ for a.e. $x \in G$, and $v_{0} \in L^{2}(G)$ are given. Then there exists a unique generalized solution of the Stefan problem (3.5).

Proof. It is clear from the above that Theorem 1 holds in our situation and that if $U$ is the solution of (4.1), then $u=K^{-1}(U)$ satisfies the generalized Stefan problem (3.5).

As with the classical Stefan problem the initial conditions (3.5c) change when $u=0$ on a set of positive measure in the initial state. When approaching initial values through $\Omega^{+}$or $\Omega^{-}, u$ and $u_{t}$ are prescribed. When approaching the initial values through $\Omega_{0}$, the water content $\xi\left(x, O^{+}\right) \in(0,1)$ must be specified.

5. The one-phase problem. The single-phase case of the Stefan problem is the description of the melting of a (partially) frozen portion $\Omega_{0}$ of the region by heat transferred from the melted portion $\Omega_{+}$and the corresponding displacement of the common interface $S$. As before, $\Omega_{+}$and $\Omega_{0}$ are the regions where $u>0$ and $u=0$, respectively, and we let $\tau, c$ and $k$ be the parameters corresponding to the melted region. While Theorem 2 can accommodate this problem, it is usual to stipulate the extra condition that $u \geq 0$ in $\Omega$. This stipulation is inconsequential for the classical Stefan problem since the classical heat equation satisfies a maximum principle, so the constraint $u \geq 0$ will be satisfied "automatically" from the nonnegativity of the initial data and boundary conditions. 
Proceeding as in Section 3 we define a generalized solution to the one phase Stefan problem as a pair of functions $u, \xi$ for which

$$
u \in W^{1, \infty}\left(0, T ; L^{2}(G)\right) \bigcap L^{\infty}\left(0, T ; H_{0}^{1}(G)\right), \quad \xi \in L^{\infty}(\Omega)
$$

$$
\frac{d}{d t}\left(\tau c u^{\prime}(t)+c u(t)+L \xi(t)\right)-k \Delta u(t)=0, \quad \text { a.e. } t \in[0, T],
$$

$$
\begin{aligned}
& u(t) \geq 0 \text { in } H_{0}^{1}(G) \quad \text { and } \quad \xi(t) \in H(u(t)) \quad \text { for all } t \in[0, T] \\
& u(0)=u_{0}, \quad\left(\tau c u^{\prime}+c u+L \xi\right)(0)=v_{0}
\end{aligned}
$$

As in the classical case, the one-phase problem (5.1) leads to a variational inequality. To see this, let $U(t)=\int_{0}^{t} u$, known as the freezing index, and set

$$
E(U)=c \tau U^{\prime \prime}(t)+c U^{\prime}(t)+\mathcal{A} U(t)-v_{0}+L .
$$

An integration in time of (5.1a) yields $E(U)=L(1-\xi(t))$. Since $\xi(t) \in H\left(U^{\prime}(t)\right)$ we obtain the variational inequality

$$
E(U) \geq 0, \quad U^{\prime}(t) \geq 0, \quad E(U)\left(U^{\prime}(t)\right)=0, \quad 0 \leq t \leq T,
$$

for a solution of (5.1a) and (5.1b). This is easily resolved by Theorem 1 . In fact, if we let $\tilde{H}$ be the maximal monotone graph given by $\tilde{H}(r)=$ $\{1\}$ for $r>0$ and $\tilde{H}(0)=(-\infty, 1]$, then we can write (5.2) formally as

$$
\frac{d}{d t}\left(\tau c u^{\prime}(t)+c u(t)+L \xi(t)\right)-k \Delta u(t)=0, \quad \text { a.e. } t \in[0, T]
$$

$$
u(t) \in H_{0}^{\prime}(G) \quad \text { and } \xi(t) \in \tilde{H}(u(t)) \quad \text { for all } t \in[0,1] .
$$

Certainly (5.3b) implies that the constraint $u(t) \geq 0$ is satisfied. If we choose $\mathcal{A}$ and $C$ as in the proof of Theorem 2 but set $\mathcal{B}(u)=c u+L \tilde{H}(u)$, we obtain from Theorem 1 the following 
Theorem 3. Assume $u_{0} \in H_{0}^{1}(G), \xi_{0} \in L^{\infty}(G)$ and $v_{0} \in L^{2}(G)$ with $\xi_{0}(x) \in H\left(u_{0}(x)\right)$ and $u_{0}(x) \geq 0$ for a.e. $x \in G$. Then there exists a unique $u \in W^{1, \infty}\left(0, T ; L^{2}(G)\right) \cap L^{\infty}\left(0, T ; H_{0}^{1}(G)\right)$ and $\xi:[0, T] \rightarrow$ $L^{2}(G)$ which satisfy $(5.3)$ and $(5.1 \mathrm{c})$.

Although Theorem 3 provides a solution of the telegraphers equation on $\Omega_{+}$, which is nonnegative on $\Omega$, we emphasize that it is not necessarily a generalized solution of (5.1). The following example illustrates the problem.

Example. Let $a>0$. Choose $\lambda=\left[\left(1+4 \tau^{2} a^{2}\right) c / 4 \tau k\right]^{1 / 2}$ and define $u(x, t)=\exp (-t / 2 \tau) \cos (a t) \sin (\lambda x)$ for $x \in G=(0, \pi / \lambda)$ in $\mathbf{R}^{1}$ and $0 \leq t \leq \pi / 2 a$. It is easy to check that $u$ satisfies (5.1) and (5.3) (with $\xi=1$ since $u \geq 0$ ) up until time $t_{0}=\pi / 2 a$. At that time $\Omega_{+}$decreases instantly to the empty set. In order to continue as a solution of (5.1a) or (5.3a) the jump condition $\left[\tau c u_{t}+L \xi\right]=0$ must be satisfied. That is,

$$
\tau c u_{t}\left(t_{0}^{+}\right)+L \xi\left(t_{0}^{+}\right)=L-\tau c a \exp \left(\frac{-\pi}{4 a \tau}\right) \sin (\lambda x) .
$$

Suppose we continue along the solution of (5.3) as given by Theorem 3. Then $u_{t}\left(t_{0}^{+}\right) \geq 0$, so

$$
\xi\left(x, t_{0}^{+}\right) \leq 1\left(\frac{\tau c a}{L}\right) \exp \left(\frac{-\pi}{4 a \tau}\right) \sin (\lambda x) .
$$

If $a$ is sufficiently large, there is an interval around $x=\pi / 2 \lambda$ in which $\xi\left(x, t_{0}^{+}\right)<0$. Thus, $(5.1 \mathrm{~b})$ is violated and more than $L$ units of latent heat were absorbed by the material. Suppose instead we continue along a solution of (5.3a) for which $\xi \geq 0$. Then (5.4) shows that for $a$ sufficiently large there is an interval around $x=\pi / 2 \lambda$ in which $u_{t}\left(x, t_{0}^{+}\right)<0$; hence, $u$ is strictly negative for some time afterward. Thus, (5.1b) is violated and the material has a region $\Omega_{-}$which is supercooled. In particular, there is no generalized solution of (5.1) with the given data.

Finally, we shall comment on two other models that have been presented and solved, in the case of one (spatial) direction, using the method of characteristics. Both models assume that the energy is 
proportional to temperature $(e=C(u)+L H(u))$ and use (1.1) to define the flux; however, they treat the free surface differently. In both instances the energy relation $[e] N_{t}=[q] \cdot N_{x}$ is imposed. $\operatorname{Li}[\mathbf{1 0}]$ obtains a second condition by requiring the temperature to be continuous while Freedman [8], following [14], imposes $[\tau q] N_{t}=[K(u)] N_{x}$. This later condition is meaningful only in the one dimensional problem and, in general, leads to discontinuous temperatures.

Like the model presented here, the above models require compatibility conditions to hold for the initial data near the free surface $(\xi \in$ $H(u)$, etc.); however, in [8] several nontrivial compatibility conditions between the initial temperature and flux are required in order to guarantee a solution. In particular, the flux, $q$, has to be suitably small; this is rather inconsistent with the plan that these nonclassical models were intended for problems involving high temperature gradients (and correspondingly large fluxes) [1-6]. Clearly, the problems with our model arise when the derivative $u^{\prime}$ is large, which will occur when large fluxes occur. There appears to be a technical problem with the model $[\mathbf{1 0}]$ for the one phase problem with zero initial temperature and prescribed temperature boundary conditions. In this situation Li demonstrates that the problem is ill posed; this is unique to that particular model.

\section{REFERENCES}

1. K. Beckurtz and K. Wirtz, Neutron physics, Springer Verlag, New York, 1964.

2. D. Bogy and P. Naghdi, On heat conduction and wave propagation in rigid solids, J. Math Phys. 11 (1970), 917-923.

3. J. Breezel and E. Nolan, Non-Fourier effects in the transmission of heat, Proc. Sixth Conf. on Thermal Conductivity, Dayton, (1966), 237-254.

4. J. Brown, D. Chung and P. Matthews, Heat pulses at low temperature, Phys. Lett. 21 (1966), 241-243.

5. C. Cattaneo, Sulla conduzione del calore, Atti Sem. Mat. Fis. Univ. Modena $3(1948 / 49), 3-21$.

6. M. Chester, Second sound in solids, Phys. Rev. 131 (1963), 2013-2015.

7. L. DeSocio and G. Gualtieri, A hyperbolic Stefan problem, Quart. Appl. Math. 41 (1983), 253-259.

8. A. Friedman and B. Hu, The Stefan problem for a hyperbolic heat equation, J. Math. Anal. Appl. 138 (1989), 249-279.

9. M. Gurtin and A. Pipkin, A general theory of heat conduction with finite wave speeds, Arch. Rational Mech. Anal. 31 (1968), 113-126. 
10. D. Li, The well-posedness of hyperbolic Stefan problem, Quart. Appl. Math., to appear.

11. J.C. Maxwell, On the dynamical theory of gases, Phil. Trans. Roy. Soc. London Ser. A 157 (1867), 49-88.

12. P. Morse and H. Feshback, Methods of theoretical physics, McGraw Hill, New York, 1953, 865.

13. M. Sadd and J. Didlake, Non-Fourier melting of a semi-infinite solid, J. Heat Trans. 99 (1977), 25-28.

14. A. Solomon, V. Alexiades, D. Wilson and J. Drake, The formulation of a hyperbolic Stefan problem, Quart. Appl. Math. 43 (1985), 295-304.

15. — , - , and J. Greenberg, A hyperbolic Stefan problem with discontinuous temperature, ORNL-6216, March 1986.

Department of Mathematics, The University of Texas at Austin, Austin, TX 78712 